\title{
Leptomeningeal cysts diagnosed by isotope cisternography
}

\author{
D. FRONT, J. M. MINDERHOUD, J. W. F. BEKS, AND L. PENNING ${ }^{1}$
}

From the Departments of Neuroradiology, Neurology, and Neurosurgery, University Hospital, Groningen, The Netherlands

SUMMARY The diagnosis of leptomeningeal cysts by isotope cisternography is described in four cases. The cysts are visualized as abnormal local collections of the radiopharmaceutical, best demonstrated 48 hours after lumbar injection. The investigation makes it possible to diagnose the cyst at an early stage, before severe clinical symptoms and changes in the bones of the skull develop. Cases of leptomeningeal cysts in various areas of the brain are described.

Isotope cisternography provides a visual assessment of flow dynamics of the cerebrospinal fluid (CSF) and has proved very valuable in the diagnosis of disturbances of CSF flow and absorption. It has recently been found to be useful also in the diagnosis of local abnormalities in CSF spaces (James et al., 1971; Front et al., 1972). In this paper four cases will be described in which leptomeningeal cysts were diagnosed by isotope cisternography.

\section{METHODS}

Radioiodinated human serum albumin (RIHSA) is used. Routinely $100 \mu \mathrm{Ci}$ are injected into the lumbar theca. The RIHSA must be freshly prepared and of high specific activity $(1 \mathrm{mg}$ albumin/100 $\mu \mathrm{Ci})$. The thyroid gland is blocked by administering three drops of Lugol's solution daily for nine days, starting one day before the investigation. Each study is performed with both a scintillation gamma camera (Pho Gamma III, Nuclear Chicago) and a rectilinear scanner (Pho Dot, Nuclear Chicago). There are several reasons for this: a preliminary study with the gamma camera gives a quick general orientation, and suggests whether it is necessary to use views and positions other than the routine anterior and lateral ones; and the rectilinear scanner gives somewhat better resolution in the plane of the collimator than does the gamma camera. Every patient is studied at 4,24 , and 48 hours after injection.

1 Reprint requests: Professor Dr. L. Penning, University Hospital, Department of Neuroradiology, Groningen, The Netherlands.

\section{CASE 1}

A 24 year old man suffered a head injury in a car accident. On examination he was subcomatose and had a mild right hemiparesis. Plain radiographs of the skull showed a depressed fracture of the lefe temporal bone, and at operation the dura mater wå found to be torn and the depressed bone fragmen penetrating the brain. The bone and the necrotio brain tissue surrounding it were excised and the dural defect was repaired. The postoperative course. was uneventful: examination at discharge frolm hospital after 14 days showed a spastic right hemiparesis and aphasia. Three months later the patient had an epileptic attack starting on the right side of his body, and this was followed by many more attacks. Nine months later the bone defect (Fig. 1) was covered with acrylic. Frequent epileptic attacks still continued despite high dosage, sometimes to toxic levels, of antiepileptic drugs. In the course of time the patient became apathetic and unable to walk, and eventually bedridden. He was readmitted two years after the accident, and RIHSA cisternography (Fig. 2) was carried out in a search for posttraumatic non-resorptive hydrocephalus. A large abnormal collection of the radiopharmaceutical was seen in the left temporoparietal region, which was thought to indicate an arachnoid cyst of the left temporoparietal region. Pneumoencephalography (Fig. 3) showed dilatation of the body and temporal horn of the left lateral ventricle in the region of the cyst. At operation a multilobulated arachnoid cyst was extirpated, and local atrophy of the brain was found in the region of the cyst. There was marked improvement after the operation: after a short while the patient could walk again, the hemiparesis was 


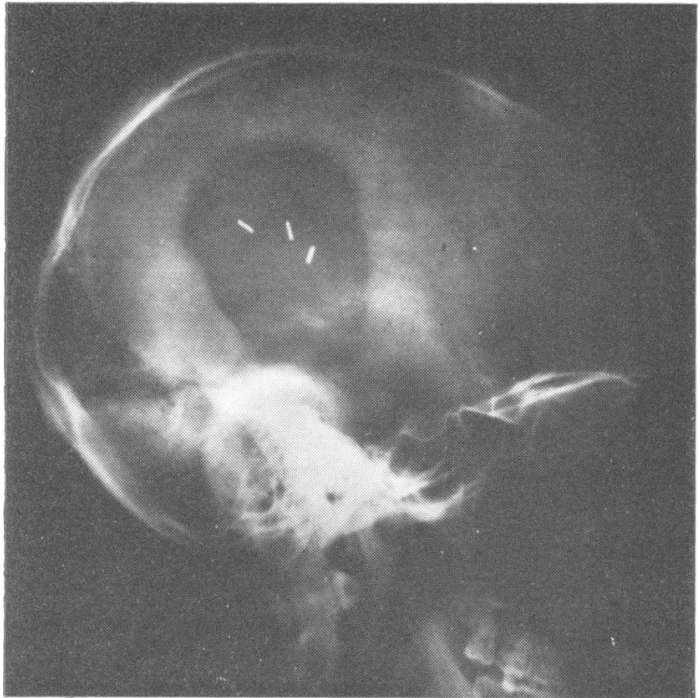

FIG. 1. Case 1. Plain radiograph of the skull. A bone defect is seen in the parietal and temporal bones.

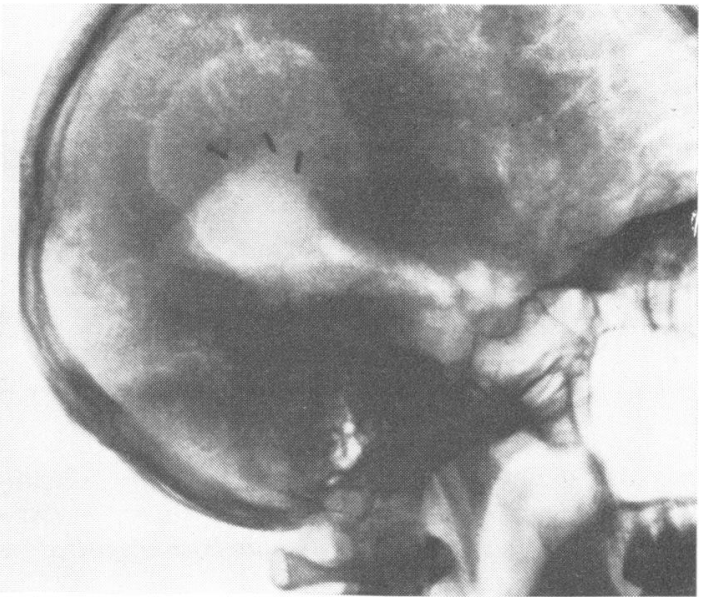

FIG. 3. Case 1. Pneumoencephalography. Right lateral decubitus, vertical $\mathrm{x}$-ray beam demonstrating dilatation of the body and temporal horn of the left lateral ventricle in the region of the cyst.

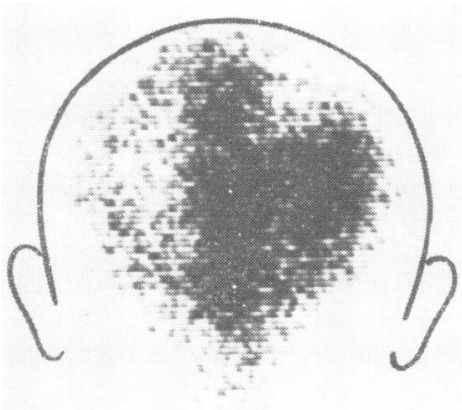

(a)

less evident, his general condition seemed to improve, and he was maintained on a low dosage of anticonvulsant drugs with no recurrence of epileptic attacks. There was no improvement, however, in the aphasia. This state remained unchanged during six months of follow-up.

\section{CASE 2}

A 70 year old woman suffered a head injury in a road accident. She was semicomatose, but there were no other abnormal neurological findings. Her cerebrospinal fluid was haemorrhagic. Over the course of a few days she regained consciousness and was then
FIG. 2. Case 1. RIHSA cisternography. Leptomeningeal cyst. (a) Anterior and (b) lateral views at 24 hours. A large parietotemporal collection of the radiopharmaceutical is seen. There is little radioactivity over the left hemisphere above the cyst. noted to be aphasic. Several electroencephalograph (EEG) recordings were taken during her hospital stay, these showed at first diffuse disturbances and then localized abnormalities over the left temporal region. Her recovery was slow and she seemed slow and apathetic. RIHSA cisternography (Fig. 4) showed an abnormal collection of the radiopharmaceutical over the left temporal region, which was best demonstrated in the 48 hour study. Pneumoencephalography (Fig. 5) showed mild dilatation of the ventricular system: the left lateral ventricle was more dilated than the right. The temporal horn of the left ventricle was disproportionately wide compared with the rest of the ventricle. After about two 


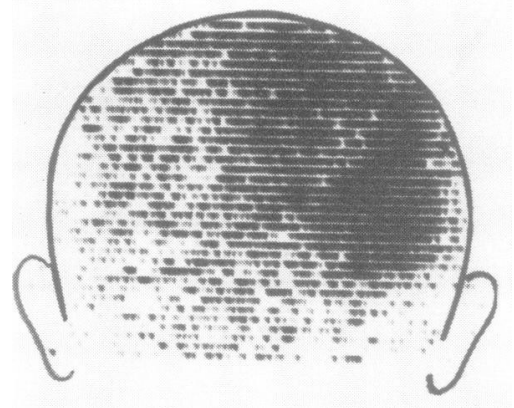

FIG. 4. Case 2. RIHSA cisternography. Leptomeningeal cyst. Anterior view at 48 hours. A large abnormal collection of the radiopharmaceutical is seen in the left temporal region.

months in hospital she began to make slow but steady improvement. She was discharged after three months in reasonable health apart from some degree of aphasia. At follow-up a year later there was no change in her condition.

\section{CASE 3}

A 16 year old girl was admitted (1962) because of complaints of headaches and visual disturbances in the right eye. Investigation showed these to be due to a pituitary tumour, and a chromophobe adenoma was removed via a left frontotemporal craniotomy. After operation the patient showed signs of hypopituitarism and was given substitution therapy, with good results. Four years later (1966) she was admitted again because of further complaints of headaches and visual disturbances, and angiography showed a large suprasellar mass extending to the right side of the sella turcica. This was further confirmed by pneumoencephalography, which also showed that the lateral ventricles were markedly dilated. At operation a large recurrent chromophobe adenoma was resected. Her postoperative course was uneventful, and substitution therapy was instituted again.

During the four years after the second operation the patient developed headaches, increasing apathy, memory disturbances, and difficulties with walking, which were not affected by increasing the dosage of hormones. Five years after the operation (1971) large amounts of clear fluid started to drip from her nose, particularly on stooping and exertion. The glucose content of the fluid was $100 \mathrm{mg} / 100 \mathrm{ml}$. RIHSA cisternography (Fig. 6) showed filling of the

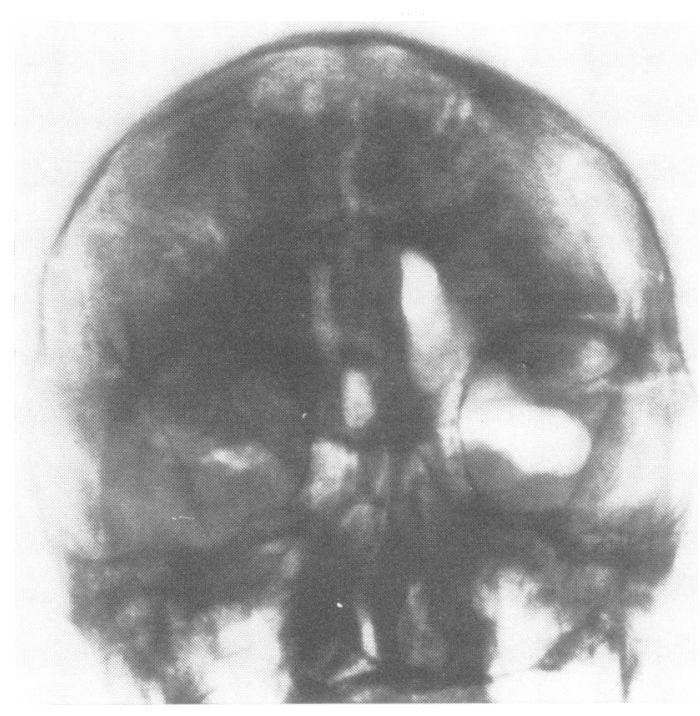

FIG. 5. Case 2. Pneumoencephalography. Right lateral decubitus, horizontal x-ray beam demonstrating dilatation of the left temporal horn.

ventricles with the radiopharmaceutical; there was no radioactivity in the subarachnoid space over the brain convexities, and an abnormal collection radioactivity was found in the basal cisterns, indic还 ting an arachnoid cyst blocking the normal passage $\overrightarrow{0}$ of cerebrospinal fluid to the convexities of the hemi- $\omega$ spheres. In view of the non-resorptive hydrocephalus the rhinorrhoea was thought to be 'high pressure rhinorrhoea'. A ventriculoatrial shunt was installed. After operation the amount of fluid dripping from the nose decreased and after a short while stopped altogether. Over a follow-up period of more than a year, there was marked improvement in her mental $\overrightarrow{0}$ state: she became more active and interested in her surroundings, and started to take care for the household. She no longer complained of headaches, and there was no recurrence of rhinorrhoea.

\section{CASE 4}

A 19 year old man suffered a head injury in a car 8 accident. On admission he was found to be semicomatose, and blood mixed with cerebrospinal fluid 을 was coming from his right nostril. Radiographs of the skull showed a fracture of the frontal bone, traversing the frontal sinus, and a fracture of the zygomatic bone. Fluid levels were seen in the $O$ sphenoidal and right maxillary sinuses, and the $N$ ethmoid sinus contained no air on the right side. N 


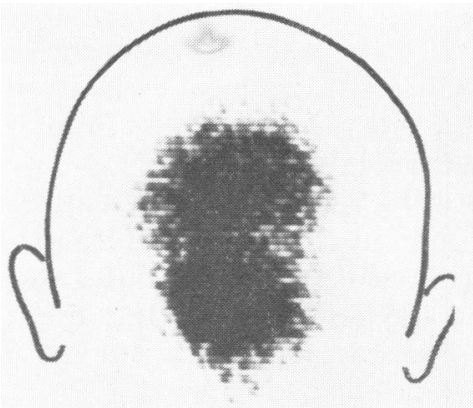

(a)

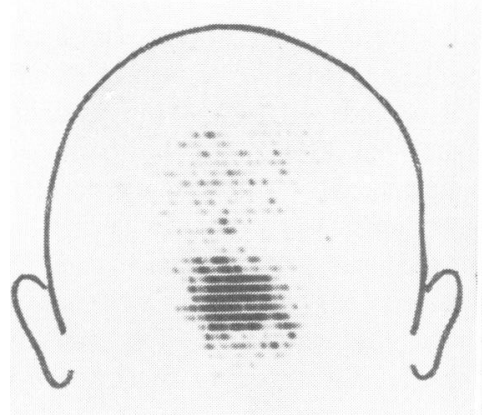

(c)

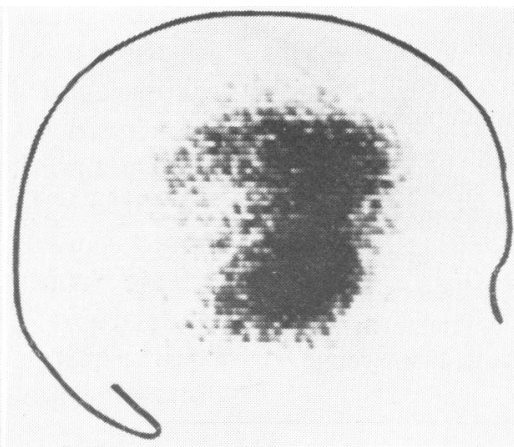

(b)

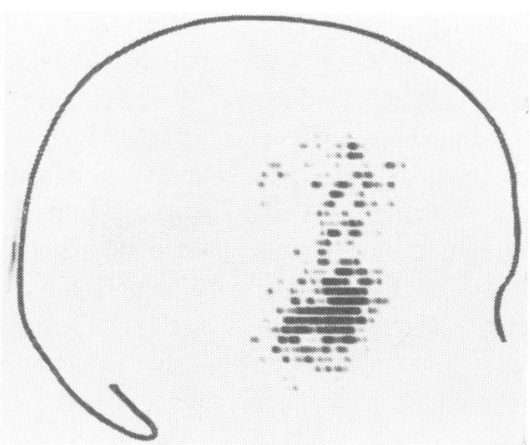

(d)

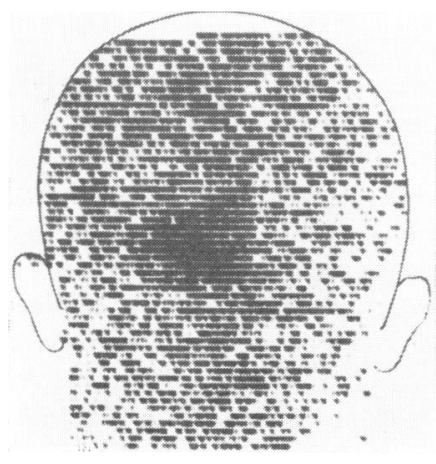

(a)

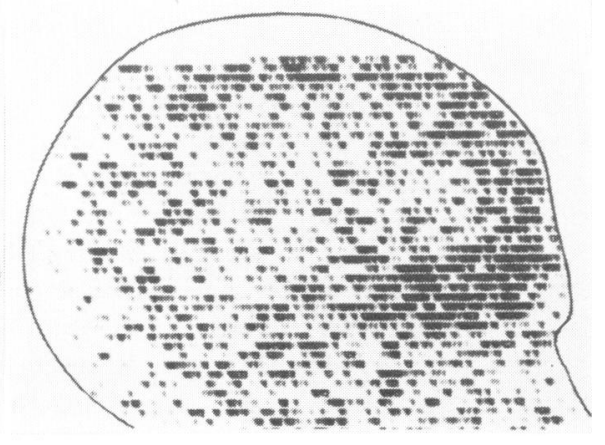

(b)
FIG. 7. Case 4. RIHSA cisternography. Leptomeningeal cyst in the floor of the anterior fossa in a case with cerebrospinal fluid rhinorrhoea. (a) Anterior and (b) lateral views at 48 hours. An abnormal local collection of the radiopharmaceutical is seen to the right of the midline in the frontobasal region.
FIG. 6. Case 3. RIHSA cisternography. Leptomingeal cyst at the base of the brain causing nonand (b) lateral views at 24 hours. An abnormal collection of RIHSA is seen in the basal cisterns. The radiopharmaventricles but not subarachnoid space over the convexities of the brain. (c) Anterior and (d) lateral views at 48 hours. The cyst is still emonstrated while only a ventricles. 
Tomograms of the right orbital region showed a fracture of the floor of the anterior fossa with dislocation of the fractured bone. In the course of a few days his level of consciousness rose and the rhinorrhoea seemed to stop. RIHSA cisternography (Fig. 7) did not reveal any trail of radioactivity, but there was an abnormal right paramedian collection in the floor of the anterior fossa. At operation a tear of the dura mater, with arachnoid adhesions forming a leptomeningeal cyst, was found. The dural defect was repaired, and the rhinorrhoea did not recur.

\section{DISCUSSION}

Leptomeningeal cysts are spaces filled with cerebrospinal fluid which result from localized areas of adhesions between the pia and the arachnoid mater (Schwartz, 1941; Taveras and Ransohoff, 1953). Traumatic cysts are caused by bleeding which irritates the meninges and causes local adhesions. They are not true cysts, since they have a communication with the subarachnoid space, but they may cause symptoms of local pressure and disturbance of CSF flow, depending on their situation. Convulsions, aphasia, and pyramidal tract signs have been described. Although known for nearly 100 years (Quain, 1855), few cases have been described (Schwartz, 1941; Cooperstock, 1946; Taveras and Ransohoff, 1953; Gruber, 1969), and this may be attributed to difficulties in diagnosis. Cysts are generally recognized by the bone changes they cause. An irregular defect in the bone, with scalloped margins, and some sclerosis at the site of an old fracture are found in traumatic cysts. However, a cyst may take months or sometimes years to grow large enough to produce any recognizable changes in the overlying bones of the calvaria. In many cases this is long after the appearance of clinical symptoms. In the cases described by Taveras and Ransohoff it took between four months and 14 years and, in the two cases described by Gruber (1969) about four months for the bone defect to develop after the accident.

Pneumoencephalography does not seem to make a very useful contribution as it does not demonstrate the cyst itself (Schwartz, 1941; Taveras and Ransohoff, 1953), but some changes may be seen. In two of the cases of Taveras and Ransohoff, and in cases 1 and 2 described here, pneumoencephalography showed local dilatation $\stackrel{\mathbb{0}}{\frac{\bar{O}}{0}}$ of the lateral ventricles in the region of the cyst. This was probably caused by local brain atrophye as a result of the injury itself and the formation of arachnoid adhesions at that site.

RIHSA, following the flow of CSF, may reach regions which air fails to fill (Di Chiro et al.,. 1964; Front et al., 1972), and as soon as adhes- $\vec{F}$ ions are formed and abnormalities appear in the CSF spaces they are demonstrated by this $\frac{0}{5}$ investigation. Bone changes appear only at a $\frac{\overline{\bar{S}}}{\bar{c}}$ later stage. Isotope cisternography thus seems to $\frac{\nabla}{\partial}$ be a useful investigation from the point of view of early diagnosis.

The reason why the radiopharmaceutical re- $\vec{\circ}$ mains in the cyst when the greater part of all $\vec{\omega}$ other radioactivity has disappeared from the $\stackrel{\mathscr{S}}{.}$ brain needs some comment. It may be explained by slow exit from the cyst to the region of normal $\dot{\omega}$ absorption, or slow absorption from the cyst itself. Yet neither of these mechanisms cap explain why in many cases the cyst exerts pre sure on its surroundings and behaves very muci, like a space occupying lesion. Pulsations of CS\$ into the small cavity of the cyst, or the bat? valve mechanism suggested by Taveras and Ransohoff-that is, CSF entering the cyst mo readily than leaving it-may be the explanatio

There was marked and immediate clinica $\vec{\varphi}$ improvement after removal of the arachnoid cyst in case 1 . In case 2 the local signs of brain damage, RIHSA cisternography, and pneumoencephalography were each very similar to those in case 1 , but the patient improved spontaneously shortly after the injury. This is not so surprising $\Phi$ as it may seem. There is growing evidence that $\stackrel{2}{\overrightarrow{2}}$ normal CSF flow may be restored after tem- $\frac{9}{3}$ porary blockage in cases with subarachnoid haemorrhage (Front and Penning, 1971) and head injuries (Front et al., 1972). It has been suggested that for a certain period of time after $\frac{5}{0}$ subarachnoid haemorrhage the arachnoid ad- $\dot{0}$ hesions may be only temporary. The essential difference between cases 1 and 2 is in the time at $\delta$ which isotope cisternography was performed. In

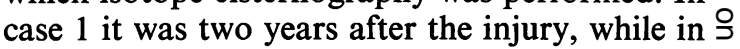
case 2 it was only two months. It may be $\frac{>}{0}$ assumed that in case 2 arachnoid adhesions at the time of the investigation were at a reversible $N$ stage, and that they disappeared later. This sug- N gests that when a cyst is diagnosed by isotope N 
cisternography shortly after trauma spontaneous recovery can be expected in certain cases. At the moment, however, experience in the diagnosis of cysts is very limited, and more reports on isotope cisternography in such cases will be necessary to select those patients needing a repeat investigation and those suitable for operation.

In case 3 the arachnoid cyst in the basal cisterns blocked the normal flow of CSF to the sylvian fissures and the subarachnoid space over the brain convexities, resulting in non-resorptive communicating hydrocephalus. This in turn caused 'high pressure' CSF rhinorrhoea (Rovit et al., 1969). After ventriculoatrial shunting the clinical state improved and the rhinorrhoea disappeared. The localization of the cyst in this case is similar to that of congenital cysts causing hydrocephalus in children (Harrison, 1971). Basal block has also been demonstrated by pneumoencephalography to be the cause of nonresorptive communicating hydrocephalus in certain cases of subarachnoid haemorrhage (Cronqvist, 1967).

Case 4 , an example of post-traumatic CSF rhinorrhoea that stopped spontaneously after a short time, showed an abnormal local collection of the RIHSA. This case is similar to one described by Di Chiro et al. (1968) and others observed by us (Front, 1971). Arachnoid adhesions in these cases result from a tear of the dura mater and leptomeninges caused by the fractured bone and local bleeding. An abnormal collection of the radiopharmaceutical in the floor of the anterior fossa may indicate a tear in the meninges in cases in which the presence of rhinorrhoea is in doubt.

\section{REFERENCES}

Cooperstock, M. (1946). Leptomeningeal cyst associated with hemiplegia and skull defect of traumatic origin. Journal of Pediatrics, 28, 488-492.

Cronqvist, S. (1967). Encephalographic changes following subarachnoid haemorrhage. British Journal of Radiology, 40, 38-42.

Di Chiro, G., Reames, P. M., and Matthews, W. B., Jr. (1964). RISA-ventriculography and RISA-cisternography. Neurology (Minneap.), 14, 185-191.

Di Chiro, G., Ommaya, A. K., Ashburn, W. L., and Briner. W. H. (1968). Isotope cisternography in the diagnosis and follow-up of cerebrospinal fluid rhinorrhea. Journal of Neurosurgery, 28, 522-529.

Front, D. (1971). Scinticisternography and scintiventriculography. Thesis, University of Groningen: Netherlands.

Front, D., and Penning, L. (1971). Angiographic assessment of ventricular enlargement in subarachnoid haemorrhage. Journal of the Neurological Sciences, 13, 1-11.

Front, D., Beks, J. W. F., Georganas, C. L., Beekhuis, H., and Penning, L. (1972). Abnormal patterns of cerebrospinal fluid flow and absorption after head injuries; diagnosis by isotope cisternography. Neuroradiology, 4, 6-13.

Front, D., Beks, J. W. F., and Penning, L. (1972). Porencephaly diagnosed by isotope cisternography. Journal of Neurology, Neurosurgery, and Psychiatry, 35, 669-675.

Gruber, F. H. (1969). Post-traumatic leptomeningeal cysts. American Journal of Roentgenology, 105, 305-307.

Harrison, M. J. G. (1971). Cerebral arachnoid cysts in children. Journal of Neurology, Neurosurgery, and Psychiatry, 34, 316-323.

James, A. E., Harbert, J. C., DeLand, F. H., McCullough, D. C., Hodges, F. J., III, and Wagner, H. N., Jr. (1971). Localized enlargement of the cerebrospinal fluid space demonstrated by cisternography. Neuroradiology, 2, 184190.

Quain, R. (1855). Large cyst from the cavity of the arachnoid. Transactions of the Pathological Society of London, 6, 8-10.

Rovit, R. L., Schechter, M. M., and Nelson, K. (1969). Spontaneous 'high-pressure cerebrospinal rhinorrhea' due to lesions obstructing flow of cerebrospinal fluid. Journal of Neurosurgery, 30, 406-412.

Schwartz, C. W. (1941). Leptomeningeal cysts from a roentgenological viewpoint. American Journal of Roentgenology, 46, 160-165.

Taveras, J. M., and Ransohoff, J. (1953). Leptomeningeal cysts of the brain following trauma with erosion of the skull. Journal of Neurosurgery, 10, 233-241. 